
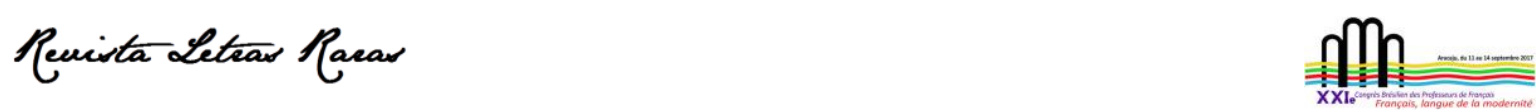

Actes du XXlème Congrès Brésilien des Professeurs De Français dans Édition spéciale de la Revue Letras Raras, 2021

ISSN : 2317-2347 - v. 10, Dossier Spécial (2021)

Todo o conteúdo da RLR está licenciado sob Creative Commons Atribuição 4.0 Internacional

\title{
De la théorie des difficultés de compréhension et/ou de traduction du FLE à l'étude de leurs manifestations : le cas de «Banque »
}

\section{Vinícius Stangherlin*}

II a fait son master en littérature française à l'UFRGS (Porto Alegre, RS, Brésil) et réalise des travaux de recherches sur les difficultés de compréhension et/ou de traduction du FLE.

(iD) https://orcid.org/0000-0002-8550-6240

\section{Robert Ponge**}

Professeur titulaire retraité de l'institut des lettres de l'UFRGS (Porto Alegre, RS, Brésil), professeur invité du centre d'études doctorales en lettres de la même université où il enseigne la littérature française et la traduction.

iD http://orcid.org/0000-0002-1078-8212

Reçu en : 05 juillet 2021. Approuvé en: 15 juillet 2021.

\section{Comment citer cet article :}

STANGHERLIN, Vinícius. PONGE, Robert. De la théorie des difficultés de compréhension et/ou de traduction du FLE à l'étude de leurs manifestations : le cas de «Banque ». Revista Letras Raras, p. 290303, n. Spécial, v. 10, nov. 2021.

\section{RÉSUMÉ}

Cette étude a pour but de présenter quelques aspects importants des travaux menés à l'UFRGS dans un laboratoire de recherche sur les difficultés de compréhension et/ou de traduction du français langue étrangère (FLE). Dans un premier moment, nous présentons nos deux grandes orientations de recherche : l'une théorique, l'autre pratique et didactique. Ensuite nous analysons l'objectif théorique (qui est aussi descriptif et classificatoire) pour, après, travailler sur quatre types de difficultés : l'homonymie, la polysémie, la paronymie et les faux amis. Nous définissons et exemplifions ces quatre genres de difficultés et faisons des commentaires sur chacun. Puis, dans un but de clarté et pour montrer les possibilités d'application de la théorie à la pratique, nous prenons le cas du mot français " banque » (établissement bancaire). II est exemplaire parce que s'y manifestent les quatre types de difficulté que nous abordons dans le présent article. À cet égard, nous montrons premièrement que l'unité linguistique « banque » possède deux homonymes, lesquels sont polysémiques. En second lieu, nous traitons de son mot sosie brésilien, banco : un faux ami dans quelques acceptions. En dernier lieu, nous signalons que "banque » possède deux paronymes en français ( « banc » et « ban »). Finalement, nous présentons quelques éléments de conclusion.

MOTS-CLÉS : Difficultés de compréhension et/ou de traduction du FLE en portugais ; Faux amis ; Homonymie ; Paronymie ; Polysémie.

$\triangle$ vinicius.stangherlin@gmail.com

$\triangle \quad$ r.ponge@ufrgs.br 

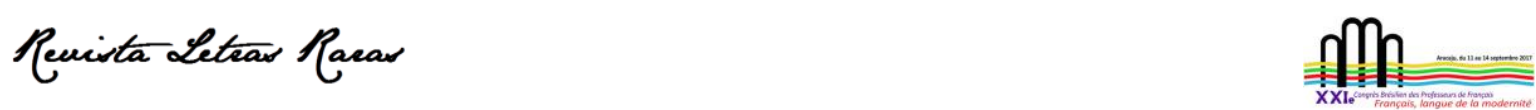

Actes du XXlème Congrès Brésilien des Professeurs De Français dans Édition spéciale de la Revue Letras Raras, 2021 ISSN : 2317-2347 - v. 10, Dossier Spécial (2021)

Todo o conteúdo da RLR está licenciado sob Creative Commons Atribuição 4.0 Internacional

\section{Introduction}

Cet article a pour but de présenter et de commenter une partie des travaux menés à l'université fédérale du Rio Grande do Sul (UFRGS) dans le laboratoire de recherche sur les difficultés de compréhension et/ou de traduction du français langue étrangère (FLE). En fonction des limites imparties à cet exposé, nous nous y restreignons à sa finalité descriptive, théorique et classificatoire : étudier les types de difficultés et leur typologie, en nous concentrant sur quatre de ceux-ci.

Quel est le plan de notre exposé ? D'abord, nous informons les deux objectifs de nos recherches et quelques concepts théoriques essentiels. Ensuite, nous détaillons l'objectif théorique et examinons quatre types de difficultés de compréhension et/ou de traduction : l'homonymie, la polysémie, la paronymie et les faux amis. Après, nous prenons le nom féminin français « banque » comme exemple et analysons les types de difficultés qu'il peut poser aux Brésiliens. Nous le faisons en étudiant son homonymie et sa polysémie, puis ses rapports de paronymie avec "ban» et "banc", deux noms masculins français. Nous considérons aussi les phénomènes qui découlent de sa ressemblance formelle avec un nom masculin de la langue portugaise, banco. Finalement, nous présentons quelques éléments de conclusion.

\section{Nos deux grandes orientations de recherche}

La finalité de nos travaux est d'aider les Brésiliens qui pratiquent le FLE à connaître et à surmonter le plus grand nombre possible de difficultés inhérentes aux processus de compréhension de cette langue et/ou de sa traduction en portugais du Brésil. Nos activités ont deux buts : 1) l'objectif théorique est d'inventorier, analyser, connaître et classifier théoriquement les principaux types de difficultés de compréhension et/ou de traduction que le français pose aux Brésiliens et 2) l'objectif pratique est d'élaborer un glossaire qui comporte le plus grand nombre possible d'articles traitant les difficultés concrètes de compréhension et/ou de traduction de la langue française. 

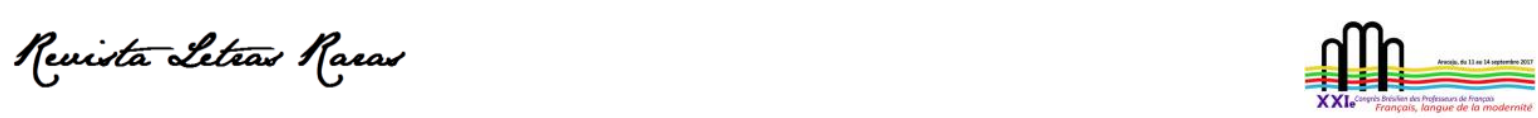

Actes du XXlème Congrès Brésilien des Professeurs De Français dans Édition spéciale de la Revue Letras Raras, 2021 ISSN : 2317-2347 - v. 10, Dossier Spécial (2021)

Todo o conteúdo da RLR está licenciado sob Creative Commons Atribuição 4.0 Internacional

Nous définissons initialement et sommairement la traduction comme l'activité consistant à « énoncer dans une autre langue (ou langue cible) ce qui a été énoncé dans une langue source, en conservant », ou plutôt en s'efforçant de conserver « les équivalences sémantiques et stylistiques » (DUBOIS, 2012, p.487).

Qu'entendons-nous par difficultés de compréhension et/ou de traduction? Ce sont les mots, les expressions ou les constructions qui suscitent couramment (ou peuvent susciter) des problèmes (obstacles, causes d'erreurs, de confusion, pièges, etc.) aux Brésiliens dans les activités de compréhension du FLE et/ou de sa traduction en portugais.

Dans nos études, nous distinguons les types de difficulté et les difficultés concrètes. Ces dernières sont les multiples occurrences de mots, syntagmes ou structures qui apparaissent dans le discours et posent des problèmes divers dans la pratique de la compréhension et/ou de la traduction. La quantité des difficultés concrètes est difficilement dénombrable, c'est une liste ouverte. Mais l'analyse des traits de chacune, dans un échantillon de celles-ci, permet de déceler l'existence de groupes de difficultés qui possèdent des caractéristiques en commun, constituant ainsi des types (ou classes ou familles ou genres) de difficultés de compréhension et/ou de traduction. Les faux amis sont un de ces ensembles, les expressions idiomatiques en sont un autre, etc.

L'objectif pratique étudie les difficultés concrètes. Pour une présentation détaillée de celui-ci, nous renvoyons à Nascimento \& Ponge (2020). Maintenant passons à...

\section{L'objectif théorique}

II a pour but d'élaborer une classification des différents types de difficultés et de définir les caractéristiques théoriques et typologiques de chacun. Comment procédons-nous ?

Initialement, nous avons commencé à organiser les bases de l'objectif théorique en consultant quelques études générales qui se penchent spécifiquement sur les types de difficultés et sur leur classification (comme MOUNIN, 1963 ; MAILLOT, 1969 ; VINAY \& DARBELNET, 1972 ; DURIEUX, 1999), et aussi des travaux ayant le même propos mais élaborés au Brésil (comme RONAI, 1976a, 1976b ; PORTINHO, 1983 ; XATARA \& OLIVEIRA, 2008).

Ensuite, de nouvelles lectures théoriques ont été faites et sont constamment proposées à la discussion de notre équipe de recherche. II s'agit de textes portant sur les domaines de la 

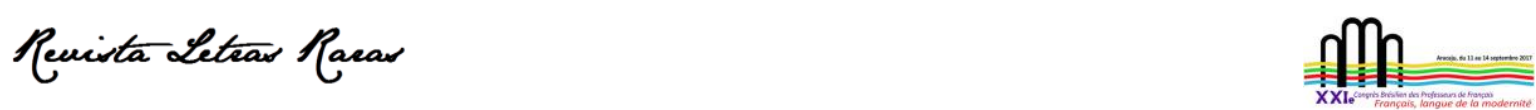

Actes du XXlème Congrès Brésilien des Professeurs De Français dans Édition spéciale de la Revue Letras Raras, 2021 ISSN : 2317-2347 - v. 10, Dossier Spécial (2021)

Todo o conteúdo da RLR está licenciado sob Creative Commons Atribuição 4.0 Internacional

compréhension du FLE, de la traduction, de la lexicographie, de la typologie des difficultés de compréhension et/ou de traduction, etc. Cela permet de réviser, corriger, enrichir, préciser les listes et les classifications de types de difficultés rencontrées. Voici une liste, encore provisoire, que nous avons établie : les faux amis, la polysémie, l'homonymie, la paronymie, la synonymie, le sens figuré des mots, les locutions idiomatiques, la connotation, le calque, les abréviations, les sigles, les noms propres, les difficultés d'ordre culturel, les divergences entre les institutions, les holophrases, entre autres.

Nous étudions également les types de difficulté en consultant la bibliographie spécifique sur le sujet. Et chaque membre de l'équipe est incité à choisir un type comme sujet personnel de recherche. II en a déjà résulté plusieurs publications (articles, mémoires) qui versent sur les faux amis (DIAS, 2007), sur l'homonymie et la polysémie (CUNHA, 2008), sur les expressions idiomatiques (SILVA, 2009), sur les sigles et les abréviations (SOARES, 2010), sur les sens figurés (STANGHERLIN, 2018).

Pour mieux comprendre la question des types de difficultés de compréhension et/ou de traduction, prenons un cas concret, le mot "banque ». Quels sont les types de difficultés que ce nom peut poser à des lusophones brésiliens ? II peut en susciter quatre, découlant de l'homonymie, de la paronymie, de la polysémie et des faux amis.

\section{L'homonymie}

Selon Dubois, il s'agit de "l'identité phonique (homophonie) ou l'identité graphique (homographie) de deux morphèmes qui n'ont pas, par ailleurs, le même sens » (2012, p.234). Neveu va dans le même sens : "l'homonymie désigne la relation de similarité de signifiant entre des formes linguistiques dont les signifiés sont différents. Cette similarité peut être tout à la fois d'ordre phonique et graphique (homophonie et homographie) » (2000, p.51).

Par exemple, car (conjonction) et car (moyen de transport) sont deux mots de même graphie et de même prononciation, mais leurs signifiés sont différents. II en va de même pour palais (de Versailles) et palais (dans la bouche) (MOUNIN, 1974, p.164, 264). Ces deux paires sont des homonymes (des homonymes homophones et homographes). Donnons un exemple en portugais : manga (fruit tropical, en français mangue) et manga (partie du vêtement, en français manche). 

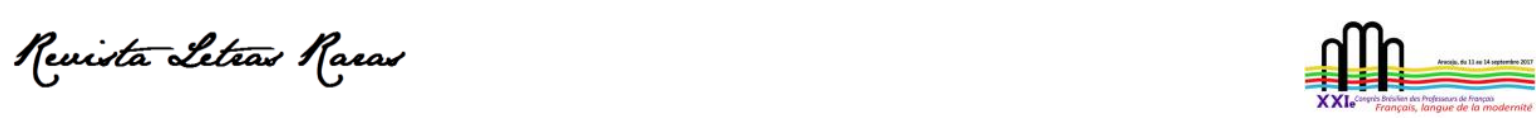

Actes du XXlème Congrès Brésilien des Professeurs De Français dans Édition spéciale de la Revue Letras Raras, 2021 ISSN : 2317-2347 - v. 10, Dossier Spécial (2021)

Todo o conteúdo da RLR está licenciado sob Creative Commons Atribuição 4.0 Internacional

Bref, l'homonymie s'avère une difficulté de compréhension du FLE quand, hors d'un contexte fort, elle peut entraîner des énoncés ambigus et causer des ennuis aux non francophones, et parfois même aux francophones : les problèmes posés par l'identité phonique et/ou graphique des formes verbales homonymes peut susciter des erreurs plus ou moins graves.

Le Dictionnaire de la linguistique dirigé par Mounin signale que «l'homonymie peut dépasser le niveau du mot» (1974, p.164). C'est le cas des rimes ou des jeux de mots et calembours - lesquels provoquent de sérieuses difficultés de traduction (comment traduire en même temps le sens et la forme ?).

Outre ce que nous avons déjà évoqué, Mounin mentionne le risque de confondre I'homonymie avec la polysémie. Analysons donc la polysémie.

\section{La polysémie}

C'est la « propriété qu'a un même signifiant de présenter plusieurs signifiés » (MOUNIN, 1974, p.264). Très proche, la définition de Neveu coïncide: «la polysémie se définit par l'existence d'une pluralité de signifiés pour un même signifiant ». Phénomène commun à toutes les langues, elle s'oppose à la monosémie (où un signifié correspond à un seul signifiant) qui n'est observée que dans le vocabulaire technique ou scientifique (NEVEU, 2000, p.52). À ce sujet, Mounin lie le degré (majeur ou mineur) de polysémie d'un mot à sa fréquence, les mots les plus fréquents étant plus polysémiques, au lieu que les moins fréquents auraient une tendance à la monosémie (1974, p.264).

Pour illustrer, passons à quelques exemples. L'adjectif «fumeux » est polysémique : dans une acception, il désigne ce "qui répand de la fumée ", dans une autre il caractérise ce " qui manque de clarté » (NEVEU, 2000, p.52). Un second exemple est « tête », substantif très polysémique en français. Son sens premier désigne la partie supérieure du corps humain, mais il possède d'autres acceptions: "faculté intellectuelle », "personne qui conçoit, dirige, organise quelque chose ", "partie antérieure d'une chose ", etc. (TLFi, 1994). Ce mot figure aussi dans un grand nombre de locutions idiomatiques formées à partir de ses diverses acceptions. Prenons maintenant le mot boca en portugais. C'est la cavité buccale, mais, par analogie, ce terme désigne aussi qualquer abertura ou fenda que lembre uma boca (AURÉLIO, 

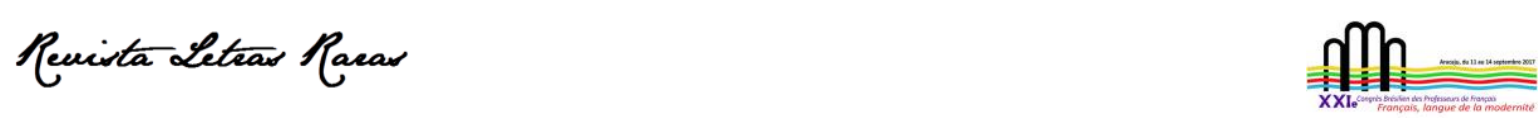

Actes du XXlème Congrès Brésilien des Professeurs De Français dans Édition spéciale de la Revue Letras Raras, 2021 ISSN : 2317-2347 - v. 10, Dossier Spécial (2021)

Todo o conteúdo da RLR está licenciado sob Creative Commons Atribuição 4.0 Internacional

1999 ; HOUAISS, 2001, toute ouverture qui donne l'idée d'une bouche) : le goulot d'une bouteille, le cratère d'un volcan, le(s) feu(x) d'une cuisinière, etc.

La polysémie peut elle aussi être un obstacle concret à la compréhension d'une langue étrangère parce qu'un destinataire étranger ne va en général connaître que les acceptions ordinaires. Au fur et à mesure qu'il avancera dans ses études ou aura plus de contact avec la langue étrangère, il lui sera possible d'approfondir ses connaissances linguistiques, mais personne (même les natifs !) ne connaîtra jamais tout le sémantisme des mots polysémiques.

Venons-en au risque de confusion entre....

\section{L'homonymie et la polysémie}

Ce sont deux phénomènes très proches. Tous les auteurs sont d'accord que la principale conséquence lexicographique de la distinction entre l'homonymie et la polysémie est que, dans l'hypothèse homonymique, le lexicographe a recours à des entrées distinctes dans le dictionnaire, tandis que dans l'hypothèse polysémique il n'y a qu'une seule entrée. Mais quels sont les critères pour définir à quelle classe appartient chaque unité lexicale ?

Pour Mounin, « la polysémie diffère de l'homonymie en cela que[, dans la polysémie,] les signifiés différents sont tout de même perçus comme présentant des traits sémantiques communs, alors que dans l'homonymie ils n'ont rien de commun ». Ce qui est juste, mais, comme nous le verrons, peut parfois s'avérer insuffisant, voire erroné. Mounin ajoute qu'un autre critère de discrimination est l'origine commune des formes : la polysémie étant attestée quand les mots ont une même étymologie et l'homonymie étant liée à des mots qui possèdent une origine différente. II s'agit toutefois d'un critère diachronique qui, dans une perspective synchronique, peut être contesté (MOUNIN, 1974, p.264-265).

Pour Neveu, le choix fait par le lexicographe entre les deux phénomènes « semblera parfois bien arbitraire et donc discutable ». Pourquoi ? Le critère étymologique peut être efficace dans cette différentiation : « si l'on a affaire à un même étymon pour deux signifiés, on formulera l'hypothèse polysémique. Si l'on a affaire à des étymons différents, on formulera l'hypothèse homonymique ». Mais si «la diversité étymologique induit l'homonymie », le contraire doit être nuancé : " l'unité de la source étymologique n'induit pas avec la même évidence la polysémie ». En d'autres termes, le critère étymologique doit être employé avec 

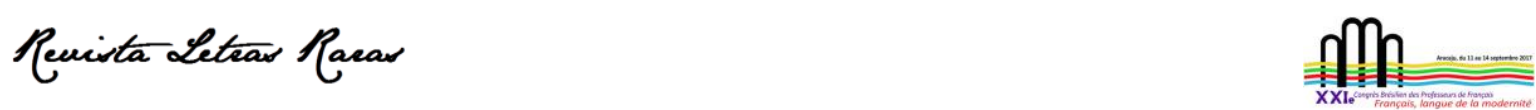

Actes du XXlème Congrès Brésilien des Professeurs De Français dans Édition spéciale de la Revue Letras Raras, 2021 ISSN : 2317-2347 - v. 10, Dossier Spécial (2021)

Todo o conteúdo da RLR está licenciado sob Creative Commons Atribuição 4.0 Internacional

prudence pour classer un mot comme polysémique, étant donné qu'il « peut présenter des signifiés dont la dérivation est si éloignée que l'approche synchronique ne pourra la détecter » (NEVEU, 2000, p.52). Par exemple, " fugue (escapade) et fugue (musique) peuvent être vus comme homonymes malgré leur origine commune » (MOUNIN, 1974, p.265). (Au sujet de I'opposition homonymie/polysémie, voir aussi DUBOIS, 2012, p.370. En portugais, voir, entre autres, le mémoire de CUNHA, 2008, et la bibliographie à laquelle il renvoie).

\section{La paronymie}

Dubois définit les paronymes comme « des mots ou des suites de mots de sens différent, mais de forme relativement voisine » (2012, p.349). Mounin n'est pas en désaccord avec cette définition, mais, intelligemment et didactiquement, offre une heureuse dénomination: les paronymes sont des quasi homonymes (« sont dits paronymes deux mots presque semblables par la forme, mais tout à fait différents par le sens, donc quasi homonymes »). C'est le cas des mots « conjoncture » (ensemble d'événements concomitants) et « conjecture » (idée formée à partir d'une probabilité ou apparence) (1974, p.249). En portugais, les mots aprender (apprendre) et apreender (saisir, appréhender), tráfego (trafic, circulation) et tráfico (trafic, contrebande) sont des paronymes.

\section{Les faux amis}

Qu'est-ce qu'un faux ami ? Le TLFi informe qu'au sens littéral un faux ami est quelqu'un qui prend abusivement l'identité ou la qualification d'ami. Il en existe un emploi figuré et, précision essentielle, « en parlant de mots de langues différentes » (TLFi, 1994), étant sous-entendu qu'ils se comportent comme des faux amis.

Selon le Dictionnaire de l'Académie française, un faux ami, au sens figuré, désigne un « mot d'une langue étrangère dont on croit deviner le sens en raison de sa ressemblance avec un mot français » (DAF, 1992). On croit, donc fausse croyance ! Les définitions du Robert/CLE et du Larousse en ligne sont presque identiques, très peu s'en faut. Le premier définit un faux ami comme « un mot d'une langue qui ressemble à un mot d'une autre langue, mais qui n'a pas le même sens » (1999, p.35). Le second ajoute seulement que la ressemblance peut 

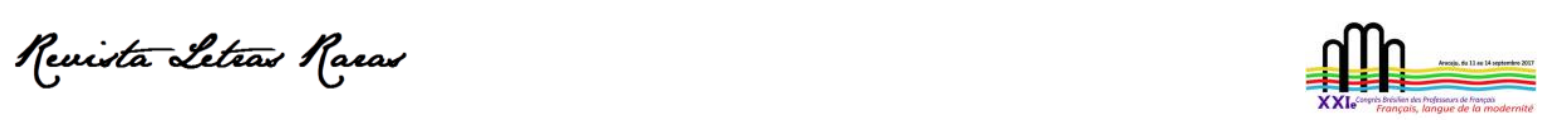

Actes du XXlème Congrès Brésilien des Professeurs De Français dans Édition spéciale de la Revue Letras Raras, 2021 ISSN : 2317-2347 - v. 10, Dossier Spécial (2021)

Todo o conteúdo da RLR está licenciado sob Creative Commons Atribuição 4.0 Internacional

être "graphique ou phonique » (LAROUSSE, 2020). Le Petit Robert explicite que la " similitude » entre les deux mots (chacun d'une langue différente) est « trompeuse », c'est-àdire sémantiquement trompeuse (2008).

Le français et le portugais possèdent beaucoup de faux amis. « Attirer » n'est pas atirar (tirer un coup de feu), mais atrair. « Pourtant » n'est pas portanto (en conséquence), mais porém. «Petite-fille » (attention au trait d'union) n'est pas menina, mais neta. "Tasse » n'est pas taça (coupe), mais xicara.

Passons maintenant à deux ouvrages de spécialistes en traduction. Commençons par Les Faux amis de Kœessler \& Derocquigny, le premier livre portant sur le sujet. Les deux auteurs soulignent qu'il s'agit d'un phénomène observé lors de « la transposition d'une langue dans une autre », lors de « la traduction ». Pourquoi ? Si I'homonymie (partielle ou non) dans une même langue ne cause pas de problèmes de ce genre, la "ressemblance verbale " peut « induire en erreur » en ce qui concerne deux systèmes linguistiques différents, car «la similitude de forme n'entraîne pas nécessairement la similitude de sens ». Le piège posé par ces mots, les faux amis, aux traducteurs est de créer des faux sens ou même des contresens : « en présence de deux mots qui, du fait de leur communauté d'origine, semblent avoir été créés à l'image l'un de l'autre, le traducteur conclut automatiquement du même au même et commet un quiproquo ». Les auteurs signalent le caractère général de ce phénomène linguistique qui - en ce qui concerne le français - intéresse surtout «toutes les langues romanes (voire les langues germaniques où figurent des vocables romans) ». Kœssler \& Derocquigny alertent qu'il faut « se méfier de ces fausses correspondances», de ces ressemblances "insidieuses " qui sont présentes dans tous les niveaux de langue et dans tous les genres de textes, même dans ceux qui sont considérés comme plus faciles à lire, à traduire (1964, p.IX-XI).

L'article "faux amis» du dictionnaire organisé par Mounin propose (à notre connaissance, il est le seul à le faire) d'appeler également faux amis « des associations de mots trop exclusives », et donne comme exemples : « fr. beau, angl[ais] beautiful, alors que : fr. un bel homme = angl. a handsome man ». Arrêtons-nous un instant sur cette deuxième acception : quelle est la cause de l'erreur de ces associations erronées ? Elles seraient «dues à une association trop fréquente par traduction » (1974, p.139), c'est-à-dire à des automatismes de traduction. Seulement de traduction? Non, nous pensons qu'en fait elles sont plus généralement dues à des automatismes d'association, de mémorisation, lesquels provoquent des 

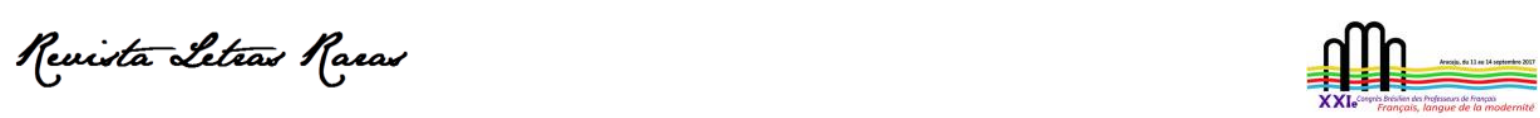

Actes du XXlème Congrès Brésilien des Professeurs De Français dans Édition spéciale de la Revue Letras Raras, 2021 ISSN : 2317-2347 - v. 10, Dossier Spécial (2021)

Todo o conteúdo da RLR está licenciado sob Creative Commons Atribuição 4.0 Internacional

erreurs, tant dans l'expression que dans la traduction, dans les situations où cet automatisme est erroné. II s'agit bien de ce que nous appelons un type de difficulté de traduction ou d'expression, type qu'il est intéressant d'étudier, mais est-ce un cas de faux amis ? Nous préférons remettre l'élucidation de cette question à un travail postérieur pour nous restreindre ici aux cas de faux amis tels que définis plus haut.

En résumé, on appelle faux amis un type de problème, de difficulté, voire de piège, qui apparaît dans la situation où (deux langues étant en rapport, en contact, en situation de bilinguisme) il existe une similitude ou apparence sémantiquement trompeuse, entre une unité ou construction linguistique de l'une des langues et une unité ou construction linguistique de l'autre langue. La «fausse correspondance » suggérée par la ressemblance entre les deux " sosies » (ces deux termes sont de Kœssler et Derocquigny, 1964, p.XI) cause le cas de faux ami, qui peut provoquer des erreurs plus ou moins graves, plus ou moins grossières chez les étudiants, mais aussi chez des gens possédant de l'expérience : des enseignants ou des traducteurs.

Les faux amis sont-ils un problème spécifique de la traduction, de « la transposition d'une langue dans une autre », comme Kœssler et Derocquigny le voulaient (1964, p.IX)? Non, il s'agit d'une difficulté de compréhension: une ressemblance formelle (totale ou partielle, graphique et/ou phonique) suggère une similitude de sens qui est trompeuse, suscitant faux sens ou contresens. Des faux amis peuvent apparaître pendant le processus traduisant, parce que la compréhension est nécessairement le premier moment de celui-ci, mais le faux ami n'est pas un problème spécifique de traduction.

Kœssler et Derocquigny font découler les occurrences de faux amis de l'étymologie, de l'existence d'une «communauté d'origine » entre les deux mots trompeurs (1964, p.X). C'est aussi la position du dictionnaire de Mounin (les faux amis sont « des mots d'étymologie et de forme semblable », 1974, p.139). En revanche, les dictionnaires de langue française consultés ne mentionnent pas l'étymologie et, d'ailleurs, ne font aucune référence à l'origine (étymologique ou non) du phénomène. Nous pensons qu'ils ont raison: l'étymologie n'a aucune pertinence pour la définition des faux amis. Qu'en pensait Paulo Rónai (1907-1992) ? Contemporain de Mounin, il a été, au Brésil, un pionnier de la réflexion sur les difficultés de compréhension et/ou de traduction. Si nous consultons ses textes sur ce sujet (voir leur analyse dans DIAS, 2007), nous observons que les définitions de faux amis y varient, on y décèle une oscillation, une hésitation (l'étymologie commune est-elle nécessaire, indispensable, définitoire ou non ?), mais 

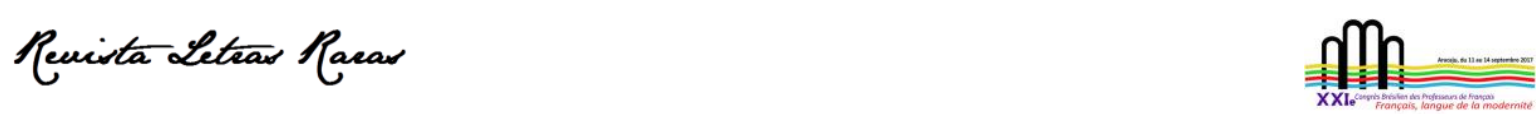

Actes du XXlème Congrès Brésilien des Professeurs De Français dans Édition spéciale de la Revue Letras Raras, 2021 ISSN : 2317-2347 - v. 10, Dossier Spécial (2021)

Todo o conteúdo da RLR está licenciado sob Creative Commons Atribuição 4.0 Internacional

somme toute il conclutà l'existence de deux sous-groupes de faux amis: avec ou sans étymologie commune (RÓNAI, in PORTINHO, 1983, p.5-7). C'est - de même, et correctement, croyons-nous - la position du TLFi qui définit les faux amis comme des mots «qui sont d'étymologie ou de forme semblable mais de sens partiellement ou totalement différent » (TLFi, 1994).

Maintenant, prenons comme exemple un mot de la langue française et examinons quels sont les types de difficultés qu'il peut poser à un pratiquant brésilien du FLE.

\section{Le mot « banque » : homonymie et polysémie}

C'est un nom féminin. Dans le Petit Robert, il possède une seule entrée, et deux dans le DAF, le TLFi, le Larousse. Comment, pourquoi ? Le Petit Robert fait silence sur des emplois spécialisés, peu fréquents que les trois autres mentionnent. C'est un danger : si l'on consulte le Petit Robert, il semble n'y avoir qu'une seule unité lexicale, alors qu'il en existe deux qui sont homonymes : «1-banque » et «2-banque ». Voyons le contenu sémantique de chacune.

«1-Banque » possède trois aires sémantiques : a) celle de l'établissement bancaire ; b) celle des jeux de cartes, de hasard où il y a des mises de fond (en monnaie ou en argent pour rire) ; c) celle des organismes, établissements ou services qui réunissent et conservent du sang (pour les transfusions), des organes (pour les greffes), du sperme, des vivres, des données, etc. (DAF, TLFi, ROBERT, LAROUSSE).

L'homonyme « 2-banque » a trois aires d'emploi spécialisé et désigne : a) (commerce ou industrie) une sorte de banc, de banquette, de table ou de comptoir ; b) (théâtre) le théâtre ou le monde des saltimbanques ; c) (géologie ou navigation) amas, levée de terre et autres matières formant un talus, un banc (DAF, TLFi, LAROUSSE).

Même si la polysémie de 1-banque peut sembler simple et ne soulever aucun problème (ou en poser peu), il reste qu'il y a deux mots différents (deux homonymes) ayant des sémantismes distincts. Donc, risques de confusion sur le plan de la compréhension : risque d'ignorer l'existence de "2-banque »; risque d'ignorer sa polysémie ; risque de confondre les deux homonymes. Risques pour qui ? Pour les francophones (qui en général méconnaissent l'existence de «2-banque»), et surtout pour les non francophones qu'ils soient étudiants, 

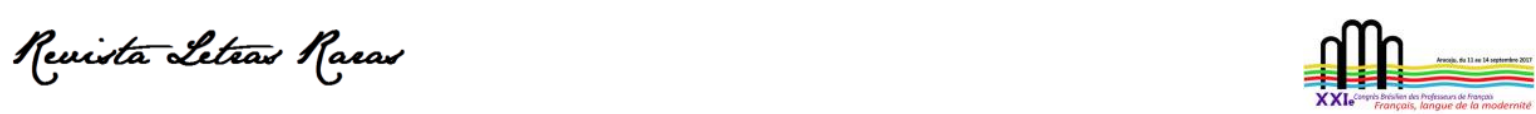

Actes du XXlème Congrès Brésilien des Professeurs De Français dans Édition spéciale de la Revue Letras Raras, 2021 ISSN : 2317-2347 - v. 10, Dossier Spécial (2021)

Todo o conteúdo da RLR está licenciado sob Creative Commons Atribuição 4.0 Internacional

enseignants, traducteurs ou autres. II est vrai que le degré de risque est peu élevé, puisque les usages de « 2-banque » sont peu fréquents, mais il n'est pas inexistant.

10 «1-Banque », « 2-banque » et leur sosie brésilien, banco

Les Brésiliens associent souvent le mot français « banque » et son sosie (ou presque sosie) de la langue portugaise, banco, car les deux mots ont une certaine ressemblance. Cette similitude d'aspect peut suggérer qu'il existerait une identité sémantique. Intuition qui a quelque chose de vrai, puisque « 1-banque » et banco réfèrent tous deux à l'établissement bancaire (1-a) et à la banque du sang, d'organes, etc. (1-c), mais partiellement seulement. Partiellement parce que banco ne réfère pas à 1-b (banca en portugais). "1-Banque » et banco sont donc des faux amis en 1-b, et des vrais amis en 1-a et 1-c.

D'autre part, la vaste polysémie du nom portugais banco inclut aussi assento estreito e duro (de madeira, pedra, ferro, cimento etc.), com ou sem apoio para os braços, ger. para mais de uma pessoa, provido ou não de encosto (AURÉLIO, 1999 ; HOUAISS, 2001), soit le type de siège: nommé "banc » en français). Or "banque » ne signifie pas «banc », sauf dans l'acception «a» de «2-banque », laquelle est spécialisée et peu fréquente. «Banque » et banco (type de siège) sont donc des faux amis, sauf en 2-a où ils sont des vrais amis. En résumé, « banque » et banco sont à la fois partiellement vrais amis et partiellement faux amis.

\section{Les homonymes « banque » et leurs paronymes}

L'unité linguistique «banque » possède deux paronymes (mots qui possèdent une graphie et/ou une prononciation proches) : «banc» (n.m.) et «ban » (n.m.). Ils ne sont pas homographes, mais les deux se prononcent de la même façon [bã] (ce sont des homonymes homophones). Quant à «banque », sa prononciation, [bãk], compte seulement un phonème de plus [k] que la leur. C'est donc un paronyme des deux autres dont il est phonétiquement proche, avec les risques de confusion que cela implique. Voyons-en les conséquences sur les plans de la compréhension, puis de la traduction.

Le contenu sémantique de «ban » est de l'ordre: a) de la proclamation publique ou solennelle d'une décision officielle, d'un futur mariage, etc.; b) de la déchéance, du 

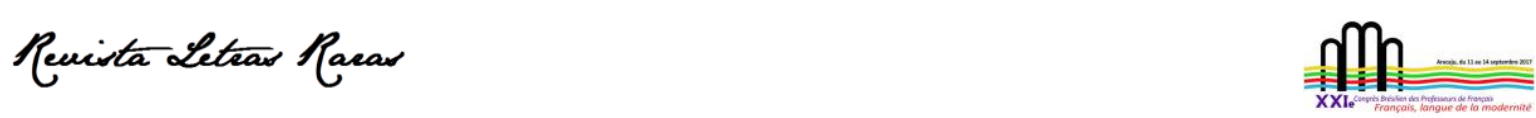

Actes du XXlème Congrès Brésilien des Professeurs De Français dans Édition spéciale de la Revue Letras Raras, 2021 ISSN : 2317-2347 - v. 10, Dossier Spécial (2021)

Todo o conteúdo da RLR está licenciado sob Creative Commons Atribuição 4.0 Internacional

bannissement, de l'exil, de la mise à l'écart ; c) (vocabulaire des temps féodaux) d'une convocation des vassaux par le suzerain (DAF, TLFi, ROBERT, LAROUSSE). Son sémantisme est donc sans rapport avec celui de "1-banque » et «2-banque », pouvant créer (en cas de confusion) un obstacle majeur à la compréhension et, a fortiori, à la traduction.

Et le contenu sémantique de "banc »? ॥ réfère à : a) siège allongé pour plusieurs personnes; b) (technique) bâti destiné à un usage artisanal ou industriel, avec ou sans instruments ou appareils de mesure, de contrôle, etc. ; c) (par extension et analogie) amas ou couche allongée de matières (sable, pierres, corail, sédiments, etc.; d) (par extension et analogie) troupe d'animaux marins (DAF, ROBERT, LAROUSSE, TLFi). Son sémantisme est sans rapport avec celui de "1-banque». Par contre (est-ce la conséquence de racines étymologiques communes ?), il existe un point de contact avec le sens «a » de "2-banque » (mais pas avec ses sens « $\mathrm{b}, \mathrm{c} \|$ ). II y a donc, sur le terrain de la compréhension, risques de confusion puisque le sémantisme des unités lexicales en question ne coïncide pas (sauf le sens « $a$ » de « 2-banque »).

Paradoxalement (et c'est hautement surprenant), les risques de confusion sont partiellement réduits sur le plan de la traduction parce que (de nouveau : est-ce la conséquence de racines étymologiques communes?): les sens «a " et " $\mathrm{C}$ " de «banc» ont souvent banco pour équivalent en portugais et son sens « $b$ » l'a parfois. Risques partiellement réduits, mais pas totalement, parce que l'équivalent du sens « $d$ » est cardume, et celui du sens «b » est plutôt bancada que banco : ce sont des sens où « banque » et banco sont toujours (ou presque) des faux amis. II serait enrichissant d'approfondir les rapports vrai amilfaux ami entre l'unité linguistique "banque» et les unités lexicales banco, banca, bancada, mais nous remettons cette réflexion à un article postérieur.

\section{Conclusion}

Nous avons ici présenté une partie de nos travaux de recherche sur les difficultés de compréhension et/ou de traduction du français. Nous nous sommes penchés surtout sur le but théorique.

Après avoir présenté celui-ci, nous avons étudié quatre types de difficultés (''homonymie, la polysémie, la paronymie et les faux amis), examiné chacun d'eux, montré quelles sont leurs 

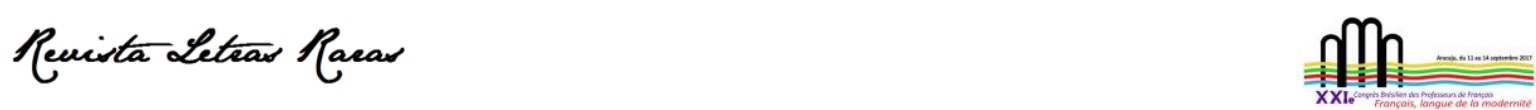

Actes du XXlème Congrès Brésilien des Professeurs De Français dans Édition spéciale de la Revue Letras Raras, 2021 ISSN : 2317-2347 - v. 10, Dossier Spécial (2021)

Todo o conteúdo da RLR está licenciado sob Creative Commons Atribuição 4.0 Internacional

caractéristiques et donné des exemples. Nous avons fait remarquer que, quand ces types de difficultés se manifestent, ils le font d'abord et avant tout comme des difficultés de compréhension. Mais l'homonymie, la paronymie et la polysémie peuvent également être cause de difficultés de traduction quand elles fournissent l'élément formel qui motive des jeux langagiers comme les jeux de mots, les calembours, et autres.

Puis, pour établir un rapport avec la didactique et avec la pratique, nous avons choisi un mot de la langue française, le nom féminin « banque » (établissement bancaire) et examiné les types de difficultés qu'il peut poser aux Brésiliens. En d'autres termes, nous avons étudié comment l'homonymie et la polysémie s'y manifestent (il y a un homonyme et il s'agit d'un mot polysémique, avec trois aires sémantiques) et quels sont les rapports de paronymie que ce mot possède avec deux noms masculins français, "banc » et "ban ». Nous avons aussi mis «banque » en parallèle avec le mot banco, nom masculin de la langue portugaise que les Brésiliens lui associent souvent, et avons mis en évidence le complexe réseau de coïncidences et de divergences entre les deux, que nous avons résumé par la formule suivante : «banque » et banco sont à la fois partiellement vrais amis et partiellement faux amis.

Finalement, nous croyons que notre article attire l'attention sur l'importance des réflexions théoriques (comme premier pas qui précède les travaux pratiques ou comme second pas qui leur succède) et qu'il permet de percevoir comment l'étude théorique typologique et classificatoire que nous menons peut contribuer positivement à l'enseignement, à l'acquisition et à la pratique du FLE ainsi qu'à la connaissance de celles-ci. Il aurait été enrichissant d'étudier les moyens de présenter ces résultats de façon plus didactique (visuellement didactique) ainsi que d'approfondir les rapports vrai ami/faux ami entre l'unité linguistique «banque » et les unités lexicales banco, banca, bancada, mais nous remettons ces réflexions à un travail postérieur.

\section{Références}

AURÉLIO: FERREIRA, A.B.H. Aurélio século XXI: dicionário da língua portuguesa. CD-ROM. Rio de Janeiro: Nova Fronteira, 1999.

CUNHA, D.A.B. A polissemia como uma dificuldade de compreensão e tradução do FLE. Monografia (Graduação), Porto Alegre: Letras/UFRGS, 2008.

DAF: Dictionnaire de l'Académie française. gème édition: 1992. Disponible sur : < http://atilf.atilf.fr/academie9.htm >. Consulté le 31 mai 2021. 

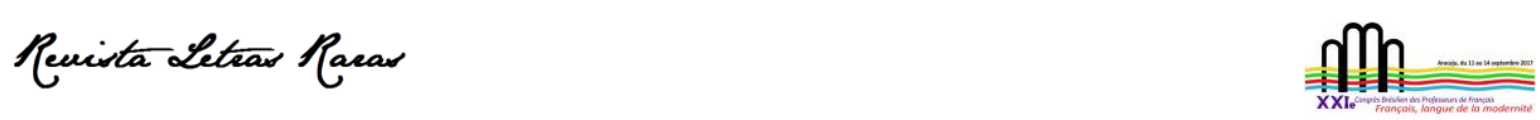

Actes du XXlème Congrès Brésilien des Professeurs De Français dans Édition spéciale de la Revue Letras Raras, 2021 ISSN : 2317-2347 - v. 10, Dossier Spécial (2021)

Todo o conteúdo da RLR está licenciado sob Creative Commons Atribuição 4.0 Internacional

DIAS, R.A. As dificuldades de compreensão e tradução do FLE e os falsos amigos. Monografia (Graduação). Porto Alegre: Letras/UFRGS, 2007.

DUBOIS, J. et alii. Dictionnaire des sciences du langage et de linguistique. Paris: Larousse, 2012.

DURIEUX, C. La difficulté en traduction. Revue des lettres et de traduction, no 5 (1999). Kaslik (Liban): Université Saint-Esprit de Kaslik (USEK). p.31-34.

HOUAISS, A. Dicionário eletrônico Houaiss da Língua Portuguesa. CD-ROM. Instituto Houaiss \& Editora Objetiva, 2001.

KOESSLER, M.; DEROCQUIGNY, J. Les Faux Amis. (1928). 6ème édition. Paris: Vuibert, 1964.

MAILLOT, J. La traduction scientifique et technique. Paris: Eyrolles, 1969.

MOUNIN, G. (Dir.). Dictionnaire de la linguistique, Paris: PUF, 1974.

MOUNIN, G. Les problèmes théoriques de la traduction, Paris: Gallimard, 1963.

NASCIMENTO, T.S.P.; PONGE, R. Quelques réflexions sur le traitement des difficultés de compréhension et/ou de traduction du français, avec trois exemples: «balade», «toit» et «parade». In: Actes du XXIlème Congrès Brésilien des Professeurs de Français. Édition spéciale de la Revue Letras Raras: Campina Grande. EDUFCG. nov. 2020, p.368-384.

NEVEU, F. Lexique des notions linguistiques. Paris: Nathan, 2000.

Petit Robert: ROBERT, P.; REY-DEBOVE, J.; REY, A. Nouveau Petit Robert, dictionnaire alphabétique et analogique de la langue française. Paris: Dictionnaires Le Robert, 1993.

PORTINHO, W.M. (org.). A tradução técnica e seus problemas. São Paulo: Álamo, 1983.

Robert/CLE: Dictionnaire du français Robert/CLE. Paris: CLE International/Le Robert, 1999.

RÓNAI, P. A tradução vivida. Rio de Janeiro: Educom, 1976a.

RÓNAI, P. Escola de tradutores. (1976b). Rio de Janeiro: Nova Fronteira, 1987.

SILVA, G.J. Um estudo sobre os idiomatismos. Monografia (Graduação). Porto Alegre: Letras/UFRGS, 2009.

SOARES, J. Les abréviations et les sigles comme difficultés de compréhension et de traduction. SEDIFRALE. Actes des 15è Sedifrale. Clef-USB/Pendrive. Rosário (Argentine): FIPF/FPFA, 2010.

STANGHERLIN, V. As dificuldades de compreensão e/ou tradução do francês: considerações sobre a designação dos usos ditos metafóricos. Monografia (Graduação). Porto Alegre: Letras/UFRGS, 2018.

TLFi - TRÉSOR DE LA LANGUE FRANÇAISE INFORMATISÉ. Nancy: ATILF-CNRS/Université de Lorraine, 1994. Disponible sur: [http://atilf.atilf.fr/]. Consulté le 31 mai 2021.

XATARA, C.M.; OLIVEIRA, W.A.L. Dicionário de falsos cognatos francês-português. $2^{\mathrm{a}}$ ed. revista e ampliada. São Paulo: Editora de Cultura, 2008.

VINAY, J-P.; DARBELNET, J. Stylistique comparée du français et de l'anglais. (1958). Édition revue et augmentée. Paris: Didier, 1972. 